\title{
Complete Absence of the Left Pericardium and Pulmonary Hypoplasia in an Adult
}

"Behnam Shakerian and Mohammad H. Mandegar

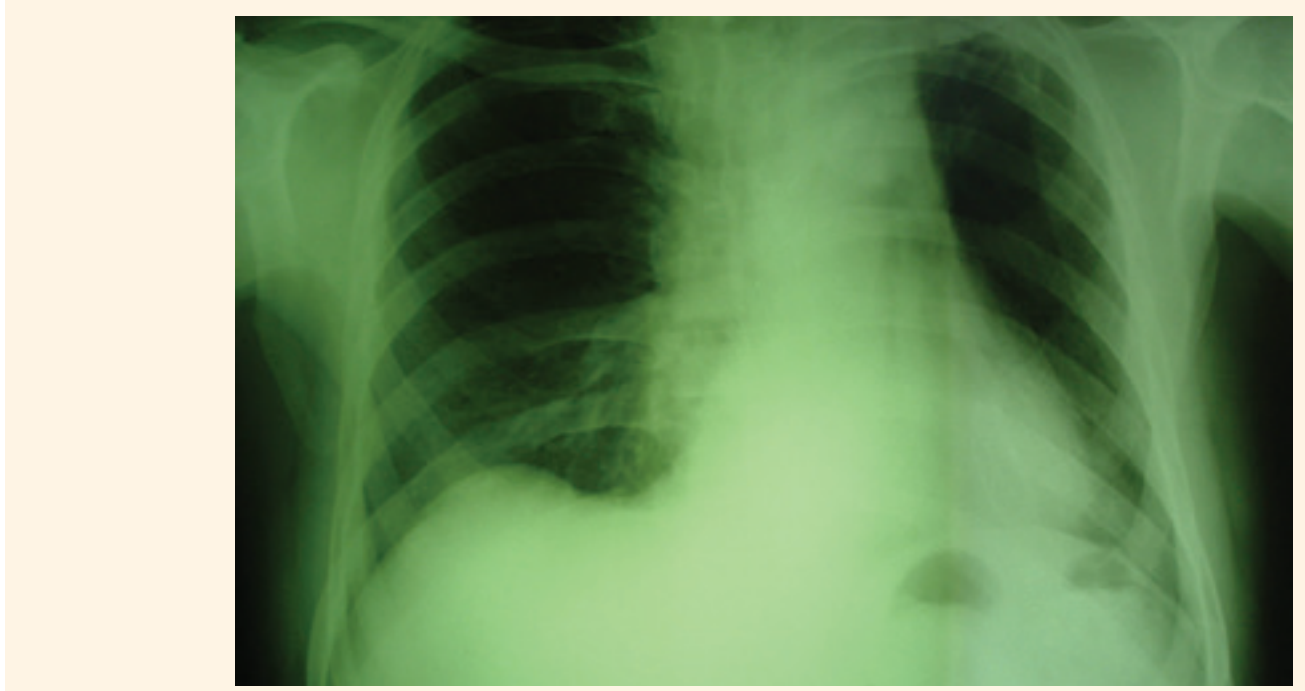

Figure 1: Chest X-ray of a 54-year-old male patient presenting to the Heart Clinic, Tehran, Iran, in 2018 showing leftward displaced heart.

A

54-YEAR-OLD MALE PATIENT PRESENTED to the Heart Clinic, Tehran, Iran, in 2018 with a history of chest pain for the previous six months. He had a relevant medical history of smoking and hyperlipidaemia. Coronary angiography revealed severe triple-vessel coronary artery disease. He was referred to the Heart Clinic for a coronary artery bypass graft (CABG). Physical examinations of the patient were normal and chest radiographic findings were normal with the exception of a cardiac silhouette that was displaced to the left [Figure 1]. Echocardiography showed a mild left ventricular dysfunction. Electrocardiography showed a normal sinus rhythm. Subsequently, the patient was scheduled to undergo CABG. During the procedure, a complete left pericardial defect and pulmonary hypoplasia were detected [Figure 2]. The left anterior descending artery was bypassed with the left internal mammary artery and a triple saphenous vein graft was done to bypass the right coronary, obtuse marginal and diagonal arteries. This defect was asymptomatic without creating pressure on the heart structures such as the left atrial appendage (LAA) or myocardium. Therefore, no intervention was performed for the defect. At the end of the surgery, a left chest drain was inserted for the defect. The patient recovered fully and was discharged on the sixth post-operative day. The patient gave his consent for publication of this report and its accompanying images.

\section{Comment}

Pericardial defects are a very rare congenital anomaly with an incidence of less than 1 in 10,000. ${ }^{1}$ Columbus first described congenital absence of the pericardium in $1559 .^{2}$ The possible embryological origins of this pericardial anomaly are not well understood, but the majority of the studies have suggested that it may be due to defective developmental retardation of the pleuropericardial membranes. $^{3}$ Approximately 30\% of patients with congenital absence of the pericardium have other congenital cardiac or pulmonary anomalies. ${ }^{4}$ Physicians should be aware of this anomaly and its associated congenital abnormalities for a correct diagnosis and treatment plan. These defects are detected incidentally during cardiothoracic operations, imaging studies or post-mortem 


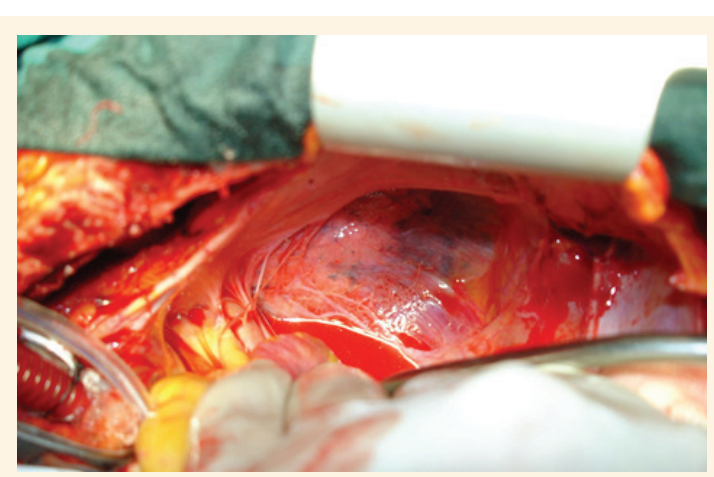

Figure 2: Intra-operative photograph of the heart showing absence of pericardium and pulmonary hypoplasia in a 54-year-old male patient presenting to the Heart Clinic, Tehran, Iran, in 2018.

examination. Pericardial defects are either partial or complete. The most common defect is absence of the left pericardium. Complete absence of the pericardium and absence of the right pericardium are less common than the absence of the left pericardium. Complete absence of the pericardium is usually asymptomatic; however, it sometimes manifests as a non-specific clinical presentation with possible symptoms including chest pain, dizziness and dyspnoea. Complete absence of the pericardium has a good prognosis. Paroxysmal exertional stabbing chest pain has been described as a potential symptom for congenital absent pericardium but the current patient did not experience this. While patients with partial absence of the left pericardium are usually also asymptomatic, there is a risk that the defect causing compression of the LAA, left atrium, coronary arteries and/or ventricles may lead to cardiac herniation. ${ }^{5}$ Echocardiography, computed tomography and magnetic resonance imaging are the most important tools for diagnosis. The treatment of absent pericardium depends on the type of the defect. ${ }^{3}$ There is usually no indication for surgery in patients with complete absence of the pericardium. ${ }^{3}$ Complications are more common in patients with partial types; therefore, interventions are necessary. ${ }^{3}$ Symptomatic or complicated cases may require pericardioplasty. Complete absence of the left pericardium leads to leftward and posterior displacement of the heart. It can be associated with hypoplasia of the left lower lobe of the lung. These combinations result in persistent left chest collections, prolonged recovery and longtime hospital stays after the operation. In patients with absent left pericardium, the left ventricle is also attached to the lateral chest wall, which may resemble pulmonary collapse or pleural effusion. Care should be taken for chest tube insertion because blind chest tube insertion could traumatise the heart. This anomaly was taken into consideration and the necessary measures for chest tube insertion were taken for the current patient.

\section{CONFLICT OF INTEREST}

The authors declare that there is no conflict of interest.

\section{References}

1. Shah AB, Kronzon I. Congenital defects of the pericardium: A review. Eur Heart J Cardiovasc Imaging 2015; 16:821-27. https://doi.org/10.1093/ehjci/jev119.

2. Saito R, Hotta F. Congenital pericardial defect associated with cardiac incarceration: Case report. Am Heart J 1980; 100:86670. https://doi.org/10.1016/0002-8703(80)90068-X.

3. Benameur N, Arous Y, Landolsi M, Chenik S, Abdallah NB, Kraiem T. Partial congenital absence of the pericardium: A case report. Braz J Cardiovasc Surg 2019; 34:633-6. https://doi. org/10.21470/1678-9741-2018-0357.

4. Kim HJ, Cho YS, Cho GY, Choi SI. Congenital absence of the pericardium. J Cardiovasc Ultrasound 2014; 22:36-9. https:// doi.org/10.4250/jcu.2014.22.1.36.

5. Han J, Xiang H, Ridley WE, Ridley LJ. Snoopy sign: Congenital absence of the left pericardium. J Med Imaging Radiat Oncol 2018; 62 Suppl 1:47. https://doi.org/10.1111/17549485.33_12785. 